\title{
Food-Borne Pathogens Associated with Natural Cheese Related Outbreaks: A Review
}

\author{
Firew Kassa \\ Ethiopian Institute of Agricultural Research, \\ Holetta Agricultural Research Center; P. O. Box 31, Holetta, Ethiopia
}

\begin{abstract}
Natural cheeses are made from pasteurized or raw milk and commonly consumed around the world. Due to their varying practices of production and/or processing, natural cheeses can be susceptible for contamination with foodborne pathogens becoming potential source of health risk for consumers. Food-borne pathogens are concerns not only for consumers but also for industries involved in production of dairy products. As in the case of all processed foods, cheese making require superb production and processing hygiene in production lines over the value chain to prevent occurrence and adverse effects of pathogens. Among others, pathogenic E. coli, Salmonella spp., S. aureus and L. monocytogenes are pathogens of the concern implicated with natural cheese related outbreaks worldwide. Therefore, this review tries to show the general characteristics of these pathogens, their brief epidemiology, source and routes of transmission, clinical significance, isolation and identification, and their prevention and control. This is aspired to serve readers and concerned audiences as important updates to contribute their parts for prevention and control of these pathogens in cheese making process.
\end{abstract}

Keywords: Natural cheese, E. coli, Salmonella, S. aureus, L. monocytogenes

DOI: $10.7176 / \mathrm{FSQM} / 97-04$

Publication date:May $31^{\text {st }} 2020$

\section{Introduction}

Cheese is one of the most popular manufactured food products of dairy origin. Natural cheese is the concentrated form of milkcontaining nutrients that can supply dietary requirement of humans and support the growth and survival of microorganisms. Cheese may also comprisenatural microflora such as lactic acid bacteria, manually added starter and non-starter cultures, and possibly contaminants including foodborne pathogens. Among others, Listeria monocytogenes, Salmonella, pathogenic Escherichia coli and Staphylococcus aureus are pathogens more often associated with cheese related foodborne disease outbreaks worldwide (FSANZ, 2009; Williams and Withers,2010). This review,brieflydescribesthese four major foodborne pathogens based on their characters, epidemiology, source and route of transmission, clinical significance, isolation and identification, and prevention and control.

\section{Listeria monocytogenes Characteristics}

Listeriais short ( 0.5 to $2 \mu \mathrm{m} \times 0.5 \mu \mathrm{m})$, gram positive, rod shaped, and psychrotrophic bacteria with rounded ends. The cells can be found singly or in short chains and sometimes in long filaments. Listeriaisalso motile at the temperatures between $20^{\circ} \mathrm{C}$ and $25^{\circ} \mathrm{C}$ with peritrichous flagella showing characteristic tumbling movement. However, it is not motile at other growth temperature conditions including optimum temperature of $37^{\circ} \mathrm{C}$. Listeriaspp. are catalase positive, oxidase negative, and they are facultative anaerobes (Liu, 2006; Liu, 2013). L. monocytogenesis a zoonotic pathogen that causesdifferent infections including mild gastroenteritis up to fatal septicemia, encephalitis, abortion or stillbirth. There are six species of Listeria known that include $L$. monocytogenes, L. ivanovii, L. welshimeri, L. grayi, L. innocula andL. seligeri. Among these, L. monocytogenes is pathogenic both for humans and animals(Liu, 2006; Liu, 2013). L. ivanovi, however, is pathogenic for animalsand rarely associated with human infection while other species are mostly nonpathogenic. In addition to the previously known six species, four novel species of Listeriaare reported recently. These include L. rocourtiae, L. marthii, L. fleischmannii, and L. weihenstephanensismaking the number of Listeria species be ten (Graves, et al., 2010;Liu, 2013).

\section{Epidemiology}

Listeria monocytogenesis widely distributed in nature, and it is a zoonotic pathogen both for animals and human.Human listeriosis has high fatality rate ofup to $30 \%$ in the risk group such as pregnant women, infant, elderly and immunocompromised adults. According to CDC report,Listeria monocytogenesis one of the major pathogens that result in an estimated 1591 illnesses, 1455 hospitalizations, and 255 deaths in the US per year (Scallan, et al., 2011). A surveillance result from France also showed that anannual incidence of 3.4 listeriosis cases occurred per 1000000 inhabitants during 2001 - 2003 (Goulet, et al., 2006). Another study from Germany indicatedthat an estimated 0.62 cases per 100000 inhabitants occur per year, and that report alsoshowed increasing 
trend of listeriosis than the previous year/s (Koch, et al., 2006). The national survey result of Japan also showed an estimated incidence of listeriosis as0.65 cases per million inhabitants that arelower than the cases in other developed countries (Okutani, et al., 2004). Other reportsfrom different regions of the world also show the distribution of L. monocytogenes all over the world (Chenal-Francisque, 2011; Blum-Menezes, 2013).

\section{Source and route of transmission}

L. monocytogenes is ubiquitous microorganism that exist in a wide range of environment including soil, water, plants, decaying vegetation, slaughter house wastes, sewage, food processing environments, etc. (Sauders, et al., 2007). This allows it potential access to foods of different origin including dairy products, meat products, vegetables, bakery products, sea foods and ready to eat foods from various sources. Because of its ubiquity, therefore, human listeriosis is believed to transmit mainly through consumption of contaminated foods (Sauders, et al., 2007; Buchholz, et al., 2001). Cheese contamination can be contracted at various stages of cheese-making process. A study conducted by Brito and colleagues showed that storage coolers might have been the principal source of contamination for cheese produced from pasteurized milk (Brito, et al., 2008). Milk as raw material and cheese making environment can also be the major sourcesofcheese contamination leading to transmission of the pathogen and consequent foodborne listeriosis incidences in the absence of proper hygienic measures.

\section{Clinical significance}

Foodborne listeriosis is a disease that affects people known as risk group that have weakened immunity. These include elderly people, newborn, pregnant women or immunocompromised people due to other diseases. Listeriosis has the clinical signs that involve febrile gastrointestinal disease with mild and flue like symptoms. The disease may also develop intomore invasive forms of listeriosis including meningitis, meningoencephalitis and septicemia in the risk groups (Buchholz, et al., 2001; Swaminathan and Gerner-Smidt, 2007; Allerberger, et al., 2010). The pathogen requires long incubation period of about 30 days where the range varies between 3 and 70 days (Swaminathan and Gerner-Smidt, 2007; Allerberger, et al., 2010).Healthy individuals with normal immunocompetence may not develop symptoms, and if they develop, it appears in a mild flu-like symptoms or mild enteric form of illness. On the risk groups, the disease is manifested in more systemic form, and it has high fatality rate of about $30 \%$ in the risk groups (Swaminathan and Gerner-Smidt, 2007). L. monocytogenesgrows in many foods including those treated with salt or food grade acids. As the organism is cold tolerant, it can also grow in those foods stored at refrigerated temperature conditions and poses a risk to the consumers. Thus, its ability to endure harsh conditions allows the pathogen to grow and reach a high number of cells in the foods as storage time extends.

\section{Isolation and identification}

Several methods have been developed and used to isolate and identify L. monocytogenes, in different specimen including food samples. Conventional bacteriological methods of ISO, FDA, and USDA-FSIS, are used as standard methods for detection of the pathogen (Donnelly and Nyachuba, 2007). These methods involve two steps that include pre-enrichment and enrichment processes in selective culture media followed by biochemical identification of suspected L. monocytogenescolonies. Since Listeriaspp. is capable of hydrolyzing esculin into esculetin, the culture methods comprise esculin and ferric ion as an important component to indicate the presence of esculin hydrolyzing bacteria.Then esculin reacts with ferric ion resulting in the formation of blackening of the media, and this indicatesthe occurrence of esculin hydrolyzing bacteria in the target sample.

The enrichment methods also contain selective agents that support the growth of $L$. monocytogenesfrom a given sample while inhibiting other microorganisms. Among these, lithium chloride is used for amplification of the pathogen in the presence of gram-negative bacteria while nalidixic acid is inhibitory for gram-negative through interference with DNA gyrase. Moreover, acriflavin, polymyxin B, and ceftazidime are used for inhibition of gram-positive cocci and to prevent the growth of gram-negative rods thatare background microflora of food and environmental samples (Donnelly and Nyachuba, 2007).

Since the start of cold enrichment techniques, a variety of detection methods have been used for isolation and identification of L. monocytogenes. However, none of the methods are best for the selective isolation and identification of the pathogen. Thus, efforts for improving the existing methods are part of the continuous hard work of scientific community. Among the existing enrichment methods, UVM broth is recommended method by USDA-FSIS for selective isolation of L. monocytogenes from meat, egg, and poultry samples. This media contains per liter of proteose peptone $(5 \mathrm{~g})$, tryptone $(5 \mathrm{~g})$, lab-lamco powder $(5 \mathrm{~g})$, yeast-extract $(5 \mathrm{~g})$, sodium chloride (20 $\mathrm{g})$, disodium phosphate 2 - hydrate $(12 \mathrm{~g})$, potassium phosphate mono-basic $(1.35 \mathrm{~g})$, esculin $(1 \mathrm{~g})$, nalidixic acid $(20 \mathrm{mg})$ and acriflavine $\mathrm{HCl}(12 \mathrm{mg})$. The medium is also called Listeria enrichment broth I (LEB I) andsimilar media with modification of its nalidixic acid content to $(25 \mathrm{mg} / \mathrm{L})$ is known as LEB II. Frazer broth is another enrichment media commonly used for isolation of L. monocytogenes. This broth is a modification of LEB II, and it contains $3 \mathrm{~g} / \mathrm{L}$ of lithium chloride in addition to the components in the LEB II (Donnelly and Nyachuba, 2007).

In addition to the enrichment broth, selective agar media are used for differential identification of the pathogen. 
Among these PALCAM and Oxford-agar are most commonly used solid media. In more recent times, chromogenic agar media have also been developed that take advantage of the specific enzyme activity that exist in $L$. monocytogenesand L. ivanovii, but not in other Listeriaspp. (Gasanov, et al., 2005). Even though the culture methods are efficient to discriminate Listeria monocytogenesselectively, food-processing companies increasingly prefer to use more rapid quality control tests. These rapid methods include those PCR-based methods, and they are known to deliver results within a short time allowing batches of foods for quick release following completion of the tests.

\section{Prevention and control}

Since $L$. monocytogenes is a ubiquitous microbe in the environment including food processing setups, it isdifficult to prevent contaminationtotally. However, implementation of appropriate hygiene and sanitation steps with good manufacturing practices and HACCP can serve for prevention and control of this pathogen. Moreover, as $L$. monocytogenesis susceptible to cooking temperature, heat treatment along with prevention of recontamination isuseful options to prevent the risk of listeriosis in foods (Huss, et al., 2000). In addition, adjusting acidification in the early stages of the process is a key factor to prevent the development of L. monocytogenes effectively with low-level contamination in raw milk cheese (Millet, et al., 2006).

In addition, different countries set the standard limit as control option so that a given food can contain the pathogen below that limit and be considered as safe for public consumption. The limits are based on the specific conditions of each country and vary from country to country. However, all the existing regulations require either absence or lower than $100 \mathrm{cfu} / \mathrm{g}$ of L. monocytogenesin cheese and other ready-to-eat food samples (Dixon, 2000; Jay, et al., 2005). For instance, USA has a strict rule of zero tolerance policy where detection of L. monocytogenesin cheese or any food sample may make the food subject to seizure. According to the regulation of European Union,however, soft cheese should be free from L. monocytogenes per $25 \mathrm{~g}$ of the sample while less than $100 \mathrm{cfu} / \mathrm{g}$ of the other cheese sample is acceptable (Dixon, 2000). Implementation of these regulations might have contributed to the reduction of outbreaks related to L. monocytogenes in the US (Jay, et al., 2005).

\section{Salmonella Characteristics}

Salmonellae are facultative anaerobic, gram-negative, smallrod-shaped bacteria $(0.7-1.5 \times 2.0-5.0 \mu \mathrm{m})$. They are member of the Enterobacteriaceae family. With the exception of $S$. Pullorum and $S$. Gallinarum, Salmonellae are motile with peritrichous flagella. They are catalase positive, oxidase negative and reduce nitrates to nitrites. Salmonella produces hydrogen sulfide, decarboxylate lysine, and they are negative toindole and urease (Bell and Kyriakides, 2002). Salmonellae are mesophilic bacteria that have a wide range of growth temperature extending between $5^{\circ} \mathrm{C}$ and $46^{\circ} \mathrm{C}$ where optimum growth temperature is around $37^{\circ} \mathrm{C}$. However, they are killed by pasteurization temperature and time. Salmonellaealso have awide range of $\mathrm{pH}$ between four and nine while optimum $\mathrm{pH}$ being around neutrality. Salmonellaedo not multiply at an $\mathrm{A}_{\mathrm{w}}$ of 0.94 , especially in combination with a $\mathrm{pH}$ of 5.5 and below but the cells survive in frozen and dried states for a long time. They can also proliferate in many foods without affecting the acceptance qualities (James, et al., 2005; Ray, 2004).

The genus Salmonella has two species namely S. enterica and S. bongori. S. entericais also subdivided into six subspecies based on their specific biochemical and genomic characteristics. As differentiation tool, Roman numbers and specific names associated with geographic location are used to designate these six subspecies. AccordinglyS. enterica subsp. enterica is designated by the Roman number (I); likewise $S$. entericasubsp. salamae (II); S. entericasubsp. arizonae (IIIa); S. entericasubsp. diarizonae (IIIb); S. enterica subsp. houtenae (IV) and $S$. entericasubsp. indica is designated by the Roman number(VI) (James, et al., 2005). Among these, S. entericasubsp. enterica is responsible for the majority of foodborne diseases.

\section{Epidemiology}

Salmonella causes bacterial foodborne disease around the world. According to the estimates, it causes 93.8 million cases of foodborne illnesses worldwide leading to 155,000 deaths each year (Majowicz, et al., 2010). Another estimate also showed that Salmonella causes about 1 million illnesses per year in the United States which result in 19336 hospitalizations and 378 deaths (Scallan, et al., 2011). A food poisoning statistics report of Ministry of Health Labor and Welfare of Japan also showed that Salmonella caused an estimated 1518 illnesses in 67 incidents occurred in various parts of the country in 2009 (MHLW, 2009). The annual burden report of different regions of the world showed that Salmonella is estimated to cause about 56300 illnesses in North Africa and Middle East.Moreover, it causes 2.5 million illnesses in Africa, 53.6 million in Asia-Pacific and Oceania, 29.8 million in South and South East Asia, 5.1 million in Europe and 2.2 million in the Americas (Majowicz, et al., 2010).

\section{Source and route of transmission}

Salmonella is transmitted through fecal-oral route mainly through ingestion of contaminated food. Consumption 
of contaminated non-animal food products, contaminated water, foods of animal origin or contact with reservoir animals are possible routes of Salmonella transmission (Majowicz, et al., 2010). In addition to this, mass production and distribution of food products disseminate pathogens rapidly to communities and occurrence of antibiotic resistance in Salmonella spp. making it a challenge to control and prevent Salmonella infections. Farm animals are the major reservoir for non-typhoid Salmonella spp. They are naturally prevalent in chickens, ducklings, sheep, goats, pigs, reptiles, amphibians, birds, pet rodents, dogs, cats, and in a variety of wild animals. Thus, consumption of foods contaminated with gastrointestinal fluid or fecal matter of these animals could result in transmission of Salmonella spp. Moreover, infections of Salmonella spp. associated with pet transmission could affect infants, and result in invasive disease and severe complications (Sánchez-Vargas, et al., 2011). Thus, though the majority of human Salmonella infections are foodborne, salmonellosis can also be acquired through contaminated drinking water, contact with infected animals and direct human-to-human transmission. Therefore, contact with a variety of animal speciesthat are infected withSalmonella, such as mammals, birds, and reptiles or consumption of contaminated food,could be a route of transmission for human infection (Majowicz, et al., 2010; Switt, et al., 2009).

\section{Clinical significance}

Salmonella spp. causes foodborne salmonellosis and result in infections that vary from mild and self-limiting gastroenteritis to sometimes systemic infections such as bacteremia. It has the incubation period of 7 to $72 \mathrm{hr}$ after ingestion of the contaminated food. The symptoms include fever, nausea, abdominal cramping, and non-bloody diarrhea thatmay tend to decrease within few hr or days. In some cases, septicemia could result as a complication of gastroenteritis leading to fatal cases in immunocompromised patients. Prolonged septicemic infections may also result in localized tissue and organ infections, especially in those previously damaged or diseased individuals. The severity and duration of symptoms may depend not only on the type and amount of pathogens ingested but also on the susceptibility of the host. The symptoms in immunocompetent hosts may typically last up to 2 to 7 days (Talbot, et al., 2006; Callaway, et al., 2008; Parry, et al., 2013; Dhanoa and Fatt, 2009).

\section{Isolation and Identification}

Regardless of the contamination level, presence of Salmonellain a given food is considered as a significant riskdue to its low infective dose. Isolation and identification of Salmonellais, conducted routinely following conventional culture techniques based on the distinct features ofSalmonellaspp. (Roberts and Greenwood, 2003; Jeniikovâ, et al., 2000). Since, food samples might have passed through different treatment methods such as cold storage or heat treatment,thecellsof the pathogen could appear in the injured state. This condition may also result in reduced recovery rate of the cell by culture method and could lead to false negative results that in its turn could posethe potential health risk tothe consumers. For this reason, isolation method usually involves pre-enrichment step to allow the recovery of injured cells. The pre-enrichment step of food sample uses non-selective media such as buffered peptone water. However, a selective RV broth is used for the enrichment step that helpsthe selective isolation of the Salmonella spp. Then the culture from RV broth is streaked on the selective differential agar media. RV broth contains malachite green and magnesium chloride that inhibits other bacteria and allows the growth of Salmonella spp.Among others, Desoxycholate agar, Salmonella-Shigella agar, modified brilliant green agar, xylose lysine Desoxycholate agar and brilliant green MacConkey agar represent selective agar media used for isolation of Salmonella. Moreover, Bismuth sulfite agar, Mannitol lysine crystal violet brilliant green agar, chromogenic agar, etc. can be used for selective isolation of Salmonella(Ray, 2004).

Salmonella spp. can be further identified by using biochemical tests. Among others, gram stains and responses to various sugar fermentation tests are commonly used methods for identification of these pathogens. In addition, ELISA and PCR-based methods are also available that has high efficiency for discrimination and get the result with shorter time than the culture methods (Ray, 2004; Roberts and Greenwood, 2003).

\section{Prevention and control}

Keeping proper sanitation measures, safe and hygienic food handling practice, and enhancing public awareness through appropriate education are some of the most important steps for the prevention of foodborne salmonellosis. Salmonellais widely present in the gastrointestinal tract of food animals and it can be shed through feces that can be the source of food contamination. Hence, prevention measures for such cases should be focused on implementing proper hygienic measures to avoid fecal contamination of foods. Among others, foods of animal origin such as eggs, poultry, and beef should be cooked properly to prevent Salmonella infection. Milk and dairy products should also be heat treated properly including pasteurization and appropriate cooking. Moreover, it is important to keep hygiene of food making environment such as kitchen surfaces and utensils usedto prevent cross contamination of other food items due to faulty practice of hygiene during household food making. Furthermore, it is important to avoid contact withgastrointestinal fluid with carcass in the slaughter houses. Avoiding the contact of infants to pet animals is also recommended as these animals are reservoirs for Salmonella. However, in the case 
of adult, it is recommended to wash hands properly following the contacts of pets (Majowicz, et al., 2010; SánchezVargas, et al., 2011).

\section{Pathogenic Escherichia coli Characteristics}

E. coli is Gram-negative, facultative anaerobic, rod-shaped bacteria belonging to the Enterobacteriaceae family. It is commensal bacteria that exist in the lower intestinal tract of human hosts and other animals in mutualistic beneficial relationship. As components of normal microflora in human intestinal tract and other warm-blooded animals, $E$. colican be detected in big number from the fecal samples. It isconsidered as harmless commensal and commonly used as indicator of fecal contamination. In addition, if its number is high, it may suggestpossible presence of enteric pathogens in foods. Though many strains of E. coli are harmless inhabitants of the gastrointestinal system, some of them cause disease in humans. The pathogenic E. coli are characterized by their serogroup, virulence genes, and production of toxins and associated disease symptoms. Accordingly, there are classes of $E$. coli pathotypes known to cause foodborne gastrointestinal disease in humans. These include enteropathogenic E. coli (EPEC), Shiga toxin-producing E. coli (STEC), enterohemorrhagic E. coli (EHEC), enteroinvasive $E$. coli (EIEC). In addition, enteroaggregative $E$. coli (EAEC), enterotoxigenic $E$. coli (ETEC), and adherent invasive E. coli (AIEC) are among the pathotypes of pathogenic E. coli (Croxen, et al., 2013; Farrokh, et al., 2013).

\section{Epidemiology}

Among pathogenic E. coli, more focus of research is given for Shiga toxin-producing E. colispecifically enterohemorrhagic E. coli (EHEC) and more data is available for this group than other pathotypes. Diseases related to the pathogenic E. coliare widely distributed all over the world. A report of CDC showsan estimated 205781 illnesses, 2429 hospitalizations and 20 deaths in the US per year due to pathogenic $E$. coli. The pathotypesinclude STEC O157, non-O157 STEC, ETEC and diarrheagenic E. coliother than STEC and ETEC (Scallan, et al., 2011). A review of Croxen and colleagues (2013) also showed that the prevalence of EPEC that goes beyond developed countries. Though it is more associated with infant diarrhea in developing countries, the problem remains in developed countries too. In the US, however, the rate related to this group of pathogens is reducing. Among all pathogenic E. coli, EHEC continues as the major concern of foodborne public health issue worldwide (Croxen, et al., 2013; Farrokh, et al., 2013). In addition to STEC O157, other groups such as O26, O45, O103, O111, O121, and $\mathrm{O} 145$ can cause hemolytic colitis and hemolytic uremic syndrome. The symptoms are apparent in infants, elderly and immunocompromised people.EHEC alone result in an estimated 112572 illness and 271 hospitalizations in the US annually (Scallan, et al., 2011). The CDC estimate also shows that the rate of EHEC incidence between 2005 and 2010 was 0.97 per 100000 inhabitants for O157 while the estimate for non-O157 was 1.10 per 100000 people. The hospitalization rate forthe same period,however, was $43 \%$ for O157 while it was $18 \%$ for non-O157 groups. The fatality rate also was twice high in the case of O157 while it was relatively low in the case of non-O157 E. coli. The incidence of O157, however, dropped by $42 \%$ in 2011 than the earlier years in the US. In Canada, Australia, and Europe the incidence reports show higher figures than those reported by CDC in the US (Croxen, et al., 2013).

\section{Source and route of transmission}

Ruminants are the major reservoir of STEC, and consumption of the food contaminated with cattle manure is the major source of the pathogen (Croxen, et al., 2013; Farrokh, et al., 2013). The main route of transmission is fecaloral route through consumption of food contaminated with the pathogen. Food contamination with cattle manure can occur through different routesand lead to the incidence of foodborne illnesses on the consumers. Direct contact with reservoir animals may also lead to human infection in the absence of appropriate hygiene. Moreover, human infection might also occur by direct contact with infected individuals especially in the places such as care centers (Croxen, et al., 2013). Thus washing hand after the contact with animals should be a regular practice before handling foods.

\section{Clinical significance}

Pathogenic E. colicause various range of clinical syndromes that involve bloody or watery diarrhea based on the pathotypes. Among these, infection of STEC can range from mild watery diarrhea to bloody diarrhea (hemolytic colitis) and may result in the development of hemolytic uremic syndrome (HUS) (Croxen, et al., 2013; Farrokh, et al., 2013). The incubation period before the onset of the symptoms couldbe about three days. Despite the variation in severity, both O157 and non-O157 STEC can cause similar symptoms including bloody diarrhea and HUS on children, elderly or immunocompromised people. EPEC,on the other hand,causes infectious diarrhea accompanied by fever, vomiting and dehydration in children under two years of age. It has a rapid onset of three-hour incubation time on human volunteers after ingesting wild-type bacteria. Other clinical features of this group include the 
intolerance for the cow's milk and failure to respond oral rehydration therapy. Clinical manifestation of EIEC is similar with that of Shigella and it is presented with bacillary dysentery and bloody diarrhea (Croxen, et al., 2013).

\section{Isolation and identification}

Pathogenic E. coli comprises several pathotypes and big number of serotypes. STEC is one of the pathotypesthat have more than 400 serotypes while only a few of them are pathogenic for humans (Croxen, et al., 2013; Farrokh, et al., 2013). Isolation methods of the pathogenic E. coli are also variable with serotypes that include culture techniques and immunological or molecular based identification methods (Vimont, et al., 2006). Since food samples might initially contain low numbers of STEC together with a high level of competing microflora, selective enrichment step is required for the isolation of STEC(Pinto, et al., 2005). Such enrichment step from inspection of processed foods could allow the recovery of injured or stressed bacterial cells due to harsh manufacturing processes (Croxen, et al., 2013; Gyles, 2007). Different enrichment protocols use varying basal broths, and diversityof selective agents are added and used with different incubation times and temperature combinations. For instance, tryptic soy broth (TSB) and E. coli (EC) broth with novobiocin are used to identify E. coli $\mathrm{O} 157 \mathrm{H} 7$ and non-O157 STEC from food samples(Croxen, et al., 2013; Gyles, 2007).

\section{Prevention and control}

Sanitizing and keeping good hygienic measure is the best way for preventing contamination of food with pathogenic $E$. coli. Since $E$. coli are highly sensitive to heat, proper temperature treatment including pasteurization or cooking foods can greatly help to prevent infection by these pathogens. In addition to heat treatment, proper refrigeration, and prevention of cross-contamination is a useful measure to control E. coli $\mathrm{O} 157 \mathrm{H} 7$ in ready-to-eat food including cheese. Therefore keeping appropriate hygienic condition of food making environment is a useful step in preventing the contamination. Moreover, personal hygiene and proper temperature treatment of foods as well as refrigeration and prevention of cross-contamination is an essential measure to prevent and control pathogenic $E$. coli in foods.

\section{Staphylococcus aureus Characteristics}

Staphylococcus aureus is gram-positive cocci that have characteristic cell arrangement of the grape-like structure. The cells appear singly, in paired cocci, or in clustered structures. It is a non-motile and non-spore forming bacteria that is facultative anaerobic (Le Loir, et al., 2003). S. aureus ferment mannitol, produce coagulase and catalase, and also produce thermostable nuclease (TNase). Itgrows in a wide temperature range that vary between 7 and $46^{\circ} \mathrm{C}$ while it optimally grows in the mesophilic temperatures between $30^{\circ} \mathrm{C}$ and $37^{\circ} \mathrm{C}$. $\mathrm{S}$. aureus also grow in relatively low Aw (0.86), low pH (4.8). Moreover, it can also growin high salt and sugar concentrations of up to $15 \%$, and in anaerobic conditions(Le Loir, et al., 2003; Kadariya, et al., 2014; Ray, 2004). Therefore, due to their ability to grow under adverse conditions, $S$. aureuscan multiply in many foods including natural cheeses. However, they are poor competitors to the natural flora that found in foods, and the presence of active starter culture inhibitstheir growth (Ray, 2004). S. aureusexists in the skin, hair, and mucosal surfaces of healthy humans and animals. In addition, anterior nares of healthy people are commonly inhabited by S. aureus on healthy carriers (Le Loir, et al., 2003;Argudín, et al., 2010). Most of the staphylococcal foodborne diseases, therefore, are thought to resultfrom consumption of contaminated foodsand due to unhygienic practices by the food handlers. Once $S$. aureusgets access to foods, it can grow and produce staphylococcal enterotoxins that are responsible for food poisoning.

\section{Epidemiology}

S. aureus causes bacterial foodborne intoxication worldwide. The data from Japanese ministry of health labor and welfare shows that $S$. aureus is one of the top most pathogens causing bacterial foodborne diseases in Japan (MHLW, 2009). S. aureus caused a total of 2525 staphylococcal food poisoning outbreaks that resulted in 59,964 illnesses, and three deaths during 20 years period of 1980 - 1999 in Japan (Shimizu, et al., 2000). Asao and colleagues (Asao, et al., 2003) also reported an extensive outbreak of the staphylococcal foodborne outbreak in Kansai area of Japan caused by the consumption of contaminated dairy products and affected 13420 people in the year 2001. S. aureus is also the second most important foodborne pathogen in France next to Salmonella. Moreover, according to another report, 86 confirmed and 173 suspected foodborne outbreaks are caused in France due to $S$. aureus among a total of 1787 foodborne illnesses reported during $2001-2003$ (Kérouanton, et al., 2007). $S$. aureusis among the major causative agents of foodborne diseases in the US where according to CDC estimate, it result in 241,148 illness, 1,064 hospitalizations and 6 deaths each year (Scallan, et al., 2011).

\section{Source and route of transmission}

S. aureusis a commensal bacterium that inhabits skin and anterior nares of humans. Among healthy humans, $20-$ 
$30 \%$ of the population is considered as permanent carriers. S. aureus also exists in the skin, hair and feathers of domestic animals and birds (Le Loir, et al., 2003; Argudín, et al., 2010). Hence, food handlers are considered as the major source offood contamination by $S$. aureus. Especially, for the heat treated foods, contamination due to the faulty handling is the main route for the transmission of the pathogen to foods. Though it is poorly competent, $S$. aureus can grow in wide varieties of foods if it gets access. Among others, milk and its products, cream filled pastries and cheeses are some of the commonly incriminated foods for staphylococcal food poisoning. Moreover, meat and meat products, poultry and egg products, salads, and bakery products, cooked meals, sandwich fillings are associated with staphylococcal food poisoning (Le Loir, et al., 2003; Ray, 2004; Argudín, et al., 2010).

\section{Clinical significance}

Consumption of one or more staphylococcal enterotoxins pre-produced in the food results in the staphylococcal food poisoning. Staphylococcal foodborne illnesses have rapid onset that normally develop within $30 \mathrm{~min}$. to $8 \mathrm{hrs}$. following ingestion of the contaminated food. The symptoms include nausea, vomiting, and abdominal cramps and with or without diarrhea(Le Loir, et al., 2003; Argudín, et al., 2010). The symptoms may also include headaches, cold sweats and rapid pulse, transient changes in blood pressure, prostration and dehydration depending on the amount of toxin ingested. Moreover, the amount of enterotoxin that causes the illness can vary depending on the susceptibility and immune condition of the individuals.However, $100-200 \mathrm{ng}$ toxin is thought to be enough to cause symptoms (Ray, 2004). A report of major Japanese outbreak estimated that about $20-100 \mathrm{ng}$ of the enterotoxin was responsible forcausing symptoms on the consumers (Asao, et al., 2003). Despite its high incidence and economic pressure on the patients, staphylococcal foodborne illness is self-limiting, and recovery from it takes one to two days. However, in rare cases it may result in complication or hospitalization (Kadariya, et al., 2014).Though it is one of the top most causes of bacterial foodborne illnesses in Japan, the death rate related to staphylococcal food poisoning is low or, no death recorded from it in recent reports (MHLW, 2009).

\section{Isolation and Identification}

$S$. aureusis isolated from food samples and other specimens following one of the standard methods such as ISO method and FDA methods among others. Enrichment in selective agar media is used for isolation of the pathogenand coagulase tests, thermo nuclease test, and other biochemical tests are used for identification (Brown, et al., 2005; Kateete, et al., 2010; Pinto, et al., 2010). Standard methods are based on the specific growth requirement of the bacteria and Baird Parker Agar and Mannitol Salt Agar with egg yolk are most commonly used agar media for isolation of $S$. aureus. Baird Parker Agar contains Tryptone and Beef Extract as major carbon and nitrogen source. Yeast Extract is another component used as essential nutrient supplement and as a main source of vitamins and growth stimulant for S. aureus. In addition to Yeast extract, the growth of the bacteria is stimulated by the presence of glycine and sodium pyruvate. Moreover, glycine, lithium chloride and potassium tellurite act as a selective agent, and egg yolk is the substrate used to detect lecithinase or lipase activity produced by $S$. aureus. Mannitol Salt Agar is another media used for selective isolation of $S$. aureus. In this medium,most other bacteria are selectively inhibited by the presence of high sodium chloride concentration. In addition to sodium chloride, mannitol is a component used as a selective agent. S. aureusferments mannitol and formation of acid due to this turns the media into yellow. Other coagulase-negative staphylococci do not ferment it and grow as red colonies. Phenol red is the $\mathrm{pH}$ indicator that is converted to yellow when the fermentation of mannitol occurs reducing the $\mathrm{pH}$ of the medium to lower than 6.8. In the absence of mannitol fermentation, however, the medium remains red. Mannitol Salt Agar also contain peptone and beef extract that supply the nutrient requirement of the bacteria including carbon, nitrogen and other essential growth factors such as trace nutrients.

Following the isolation of $S$. aureus on selective media, identification is achieved through respective biochemical analysis specific to this pathogen that includes colony morphology and gram staining. Moreover, coagulase test, latex agglutination test, DNase and thermo nuclease test, and tests based on commercial biochemical kits are used for identification. Furthermore, PCR-based tests of molecular methods targeting specific genetic markers of $S$. aureus can be used for identification (Brown, et al., 2005). Among others, coagulase test is most commonly used test for identification of $S$. aureus in routine laboratory activities (Kateete, et al., 2010). There are two types of coagulase tests used for screening and identifying $S$. aureus. These include slide coagulase test (SCT) and tube coagulase test (TCT). Bound coagulase produced by the $S$. aureus and also known as clumping factor can be identified by using slide coagulase test. This test crosslinks $\alpha$ and $\beta$ chains of fibrinogen in rabbit plasma to produce fibrin clots that deposits on the cell wall of $S$. aureus. As a result, every cell of $S$. aureus sticks to each other and show the resultant clumping of cells (Kateete, et al., 2010; Katz, 2013). Tube coagulase test, on the other hand, is used to identify the presence of free extracellular coagulase. This clots rabbit plasma in the absence of calcium and the test is the standard method for routine identification of S. aureus.

\section{Virulence factors}

$S$. aureus has diverse virulence factors thatinclude adhesions and extracellular proteins; and that enable them to 
colonize and cause various kind of illnesses (Pereira, et al., 2009). Among others, staphylococcal enterotoxins are the most important virulence factors related to the food poisoning illnesses. Classically five staphylococcal enterotoxins (SEA, SEB, SEC, SED, and SEE) are known for their involvement in staphylococcal food poisoning incidences. However, at present there are about 22 staphylococcal enterotoxins identified among which some of them are known to be emetic and recognized for their involvement in foodborne outbreaks (Argudín, et al., 2010; Hennekinne, et al., 2010). Therefore, those having emetic activities are designated as staphylococcal enterotoxins. However, the other group either lack emetic activity or not yet examined for such activity and they are designated as staphylococcal enterotoxin like super-antigens (Lina, et al., 2004).

\section{Prevention and control}

Since $S$. aureus can contaminate foods mainly due to faulty hygienic practice, improving overall production hygiene is the most important way to prevent contamination. In addition, since $S$. aureus is highly susceptible to heat treatment appropriate temperature treatment including pasteurization or cooking is highly important preventive measure. Moreover, personal hygiene thatinvolves washing hands and equipment properly, wearing masks, wearing hair nets, using separate equipment among others are useful for prevention of contamination (Kadariya, 2014).Furthermore, as $S$. aureus can't grow at that temperature, storing natural cheese at the temperature lower than $5^{\circ} \mathrm{C}$ may help to prevent and control the pathogen and possible toxin production(Kadariya, 2014; Ray, 2004).

\section{Conclusion}

Natural cheese is the concentrated form of milk containing nutrients that can supply dietary requirement of humans. Among others, Listeria monocytogenes, Salmonella, pathogenic Escherichia coli and Staphylococcus aureus are pathogens often associated with cheese related foodborne disease outbreaks worldwide. L. monocytogenes is widely distributed in nature, and it is a zoonotic pathogen. Human listeriosis has high fatality rate of up to $30 \%$ in pregnant women, infant, elderly and immunocompromised adults. Listeriosis has the clinical signs that involve febrile gastrointestinal disease with mild and flue like symptoms. The disease may also develop into more invasive forms of listeriosis including meningitis, meningoencephalitis and septicemia in the risk groups. Implementation of appropriate hygiene and sanitation steps with good manufacturing practices and HACCP can serve for prevention and control of this pathogen.

Salmonella is facultative anaerobic, gram-negative, small rod-shaped bacteria of Enterobacteriaceae family. It is transmitted through fecal-oral route mainly when contaminated food products are ingested. Salmonella causes foodborne salmonellosis and result in infections that vary from mild and self-limiting gastroenteritis to sometimes systemic infections such as bacteremia. The symptoms include fever; nausea, abdominal cramping, and nonbloody diarrhea that may tend to decrease within few hr or days. Regardless of the contamination level, presence of Salmonella in a given food is considered as a significant risk due to its low infective dose. Keeping proper sanitation measures, safe and hygienic food handling practice, and enhancing public awareness through appropriate education are some of the most important steps for the prevention of foodborne salmonellosis.

E. coli is Gram-negative, facultative anaerobic, rod-shaped bacteria belonging to the Enterobacteriaceae family. It is commensal bacteria that exist in the lower intestinal tract of human hosts and other animals. E. coli can be detected in big number from the fecal samples. Diseases related to the pathogenic E. coli are widely distributed all over the world. Ruminants are the major reservoir of STEC, and consumption of the food contaminated with cattle manure is the major source of the pathogen as it mainly transmits through fecal-oral route. Pathogenic E. coli cause various range of clinical syndromes that involve bloody or watery diarrhea based on the pathotypes. Sanitizing and keeping good hygienic measure is the best way for preventing contamination of food with pathogenic $E$. coli. Since $E$. coli are highly sensitive to heat, proper temperature treatment including pasteurization or cooking foods can greatly help to prevent infection by these pathogens.

S. aureus is gram-positive cocci that have characteristic cell arrangement of the grape-like structure. It is a non-motile and non-spore forming, facultative anaerobic bacteria. $S$. aureus causes bacterial foodborne intoxication worldwide. S. aureus is a commensal bacterium that inhabits skin and anterior nares of humans. Among healthy humans, $20-30 \%$ of the population is considered as permanent carriers. It also exists in the skin, hair and feathers of domestic animals and birds. Consumption of one or more staphylococcal enterotoxins preproduced in the food results in the staphylococcal food poisoning. Staphylococcal foodborne illnesses have rapid onset that normally develop within $30 \mathrm{~min}$ to $8 \mathrm{hrs}$. The symptoms include nausea, vomiting, and abdominal cramps and with or without diarrhea. Since $S$. aureus can contaminate foods mainly due to faulty hygienic practice, improving overall production hygiene is the most important way to prevent contamination. In addition, as $S$. aureus is highly susceptible to heat treatment appropriate temperature treatment including pasteurization or cooking is highly important preventive measure. 


\section{Acknowledgement}

Obihiro University of Agriculture and Veterinary Medicine and Ethiopian Institute of Agricultural Research are highly acknowledged for financial support and study arrangements.

\section{References}

Allerberger F, Wagner M: Listeriosis: A resurgent foodborne infection. Clin Microbiol Infect 2010, 16:16-23.

Argudín MÁ, Mendoza MC, Rodicio MR: Food Poisoning and Staphylococcus aureus Enterotoxins. Toxins (Basel) 2010, 2:1751-1773.

Asao T, Kumeda Y, Kawai T, Shibata T, Oda H, Haruki K, Nakazawa H, Kozaki S: An extensive outbreak of staphylococcal food poisoning due to low-fat milk in Japan: estimation of enterotoxin A in the incriminated milk and powdered skim milk.Epidemiol Infect 2003, 130:33-40.

Bell C, Kyriakides A: SALMONELLA A Practical Approach to the Organism and Its Control in Foods. Oxford: Blackwell Science; 2002:1-25.

Blum-Menezes D, Deliberalli I, Bittencourt NC, Do Couto CAT, Barbosa LN, Dos Santos AM, Pinto GG: Listeriosis in the far South of Brazil: Neglected infection?Rev Soc Bras Med Trop 2013, 46(January):381383.

Brito JRF, Santos EMP, Arcuri EF, Lange CC, Brito M a VP, Souza GN, Cerqueira MMPO, Beitran JMS, Call JE, Liu Y, Porto-Fett ACS, Luchansky JB: Retail survey of Brazilian milk and Minas frescal cheese and a contaminated dairy plant to establish prevalence, relatedness, and sources of Listeria monocytogenes isolates. Appl Environ Microbiol 2008, 74:4954-4961.

Brown DFJ, Edwards DI, Hawkey PM, Morrison D, Ridgway GL, Towner KJ, Wren MWD: Guidelines for the laboratory diagnosis and susceptibility testing of methicillin-resistant Staphylococcus aureus (MRSA). $J$ Antimicrob Chemother 2005, 56(November):1000-1018.

Buchholz U, Mascola L: Transmission, Pathogenesis, and Epidemiology of Listeria monocytogenes. Infect Dis Clin Pract 2001, 10:34-41.

Callaway TR, Edrington TS, Anderson RC, Byrd J a., Nisbet DJ: Gastrointestinal microbial ecology and the safety of our food supply as related to Salmonella.J Anim Sci 2008, 86:163-172.

Chenal-Francisque V: Worldwide Distribution of Major Clones of Listeria monocytogenes. Emerg Infect Dis 2011, 17:1110-1112

Croxen M a., Law RJ, Scholz R, Keeney KM, Wlodarska M, Finlay BB: Recent advances in understanding enteric pathogenic Escherichia coli. Clin Microbiol Rev 2013, 26:822-880.

Dhanoa A, Fatt QK: Non-typhoidal Salmonella bacteraemia: epidemiology, clinical characteristics and its' association with severe immunosuppression.Ann Clin Microbiol Antimicrob 2009, 8:15.

Dixon PH: European Systems for the Safe Production of Raw Milk Cheese. 2000:59.

Donnelly CW, Nyachuba DG: Conventional methods to detect and isolate Listeria monocytogenes. In Listeria, listeriosis, and food safety. 3rd edition. Edited by Ryser ET, Marth EH. Boca Raton: CRC; 2007:218-249.

Farrokh C, Jordan K, Auvray F, Glass K, Oppegaard H, Raynaud S, Thevenot D, Condron R, De Reu K, Govaris A, Heggum K, Heyndrickx M, Hummerjohann J, Lindsay D, Miszczycha S, Moussiegt S, Verstraete K, Cerf O: Review of Shiga-toxin-producing Escherichia coli (STEC) and their significance in dairy production. Int J Food Microbiol 2013, 162:190-212.

FSANZ: Food Standards Australia Newzealand - Microbiological Risk Assessment of Raw Milk Cheeses. 2009 (December 2009).

Gasanov U, Hughes D, Hansbro PM: Methods for the isolation and identification of Listeria spp. and Listeria monocytogenes: A review. FEMS Microbiol Rev 2005, 29:851-875.

Goulet V, Jacquet C, Martin P, Vaillant V, Laurent E, Valk H de: Surveillance of Human Listeriosis in France, 2001-2003. Eurosurveillance 2006, 11:79-81.

Graves LM, Helsel LO, Steigerwalt AG, Morey RE, Daneshvar MI, Roof SE, Orsi RH, Fortes ED, Milillo SR, Den Bakker HC, Wiedmann M, Swaminathan B, Sauders BD: Listeria marthii sp. nov., isolated from the natural environment, Finger Lakes National Forest. Int J Syst Evol Microbiol 2010, 60:1280-1288.

Gyles CL: Shiga toxin-producing Escherichia coli: an overview.J Anim Sci 2007, 85:E48-E62.

Hennekinne JA, Ostyn A, Guillier F, Herbin S, Prufer AL, Dragacci S: How should staphylococcal food poisoning outbreaks be characterized?Toxins (Basel) 2010, 2:2106-2116.

Huss HH, Jørgensen LV, Vogel BF: Control options for Listeria monocytogenes in seafoods. Int J Food Microbiol 2000, 62:267-274

James MJ, Loessner MJ, Golden DA: Foodborne Gastroenteritis Caused by Salmonella and Shigella. In Modern Food Microbiology. 7th edition. New York: Springer; 2005:619-630.

Jay JM, Loessner MJ, Golden DA: Foodborne Listeriosis. In Modern Food Microbiology. 7th edition. New York: Springer; 2005:591-617.

Jenïkovâ G, Pazlarovâ J, Demnerovâ K: Detection of Salmonella in food samples by the combination of 
immunomagnetic separation and PCR assay. Int Microbiol 2000, 3:225-229.

Kadariya J, Smith TC, Thapaliya D: Staphylococcus aureus and staphylococcal food-borne disease: an ongoing challenge in public health.Biomed Res Int 2014, 2014:827965.

Kateete DP, Kimani CN, Katabazi F a, Okeng A, Okee MS, Nanteza A, Joloba ML, Najjuka FC: Identification of Staphylococcus aureus: DNase and Mannitol salt agar improve the efficiency of the tube coagulase test.Ann Clin Microbiol Antimicrob 2010, 9:23.

Katz DS: Coagulase Test Protocol - Library. American Society for Microbiology, ASM MicrobeLibrary 2013.

Kérouanton A., Hennekinne J A., Letertre C, Petit L, Chesneau O, Brisabois a., De Buyser ML: Characterization of Staphylococcus aureus strains associated with food poisoning outbreaks in France. Int J Food Microbiol 2007, 115:369-375.

Koch J, Stark K: Significant Increase of Listeriosis in Germany - Epidemiological Patterns 2001 - 2005. Eurosurveillance 2006, 11:85-88.

Le Loir Y, Baron F, Gautier M: Staphylococcus aureus and food poisoning. Genet Mol Res 2003, 2:63-76.

Lina G, Bohach G a, Nair SP, Hiramatsu K, Jouvin-Marche E, Mariuzza R: Standard nomenclature for the superantigens expressed by Staphylococcus.J Infect Dis 2004, 189:2334-2336.

Liu D: Identification, subtyping and virulence determination of Listeria monocytogenes, an important foodborne pathogen. J Med Microbiol 2006, 55:645-659.

Liu D: Molecular approaches to the identification of pathogenic and nonpathogenic listeriae.Microbiol insights 2013, 6:59-69.

Majowicz SE, Musto J, Scallan E, Angulo FJ, Kirk M, O’Brien SJ, Jones TF, Fazil A, Hoekstra RM: The global burden of nontyphoidal Salmonella gastroenteritis.Clin Infect Dis 2010, 50:882-889.

MHLW: Japanese Ministry of Health, Labor and Welfare; Food Poisoning Statistics, 2009:0-40.

Millet L, Saubusse M, Didienne R, Tessier L, Montel MC: Control of Listeria monocytogenes in raw-milk cheeses. Int J Food Microbiol 2006, 108:105-114.

Okutani A, Okada Y, Yamamoto S, Igimi S: Nationwide survey of human Listeria monocytogenes infection in Japan.Epidemiol Infect 2004, 132:769-772.

Parry CM, Thomas S, Aspinall EJ, Cooke RPD, Rogerson SJ, Harries AD, Beeching NJ: A retrospective study of secondary bacteraemia in hospitalised adults with community acquired non-typhoidal Salmonella gastroenteritis.BMC Infect Dis 2013, 13:107.

Pereira V, Lopes C, Castro a., Silva J, Gibbs P, Teixeira P: Characterization for enterotoxin production, virulence factors, and antibiotic susceptibility of Staphylococcus aureus isolates from various foods in Portugal. Food Microbiol 2009, 26:278-282.

Pinto B, Chenoll E, Aznar R: Identification and typing of food-borne Staphylococcus aureus by PCR-based techniques. Syst Appl Microbiol 2005, 28:340-352.

Ray B: Fundamental Food Microbiology: Salmonellosis by Salmonella. 2004:625.

Ray B: Fundamental Food Microbiology: Staphylococcal Intoxication. 2004:625.

Roberts D, Greenwood M: Practical Food Microbiology. 3rd edition. Malden: Blackwell; 2003:294.

Sánchez-Vargas FM, Abu-El-Haija M A., Gómez-Duarte OG: Salmonella infections: An update on epidemiology, management, and prevention. Travel Med Infect Dis 2011, 9:263-277.

Sauders BD, Wiedmann M: Ecology of Listeria Species and L. monocytogenes in the Natural Environment. In Listeria, Listeriosis and Food Safety. 3rd edition. Edited by Ryser ET, Marth EH. Boca Raton: CRC; 2007:21-44

Scallan E, Hoekstra RM, Angulo FJ, Tauxe R V., Widdowson MA, Roy SL, Jones JL, Griffin PM: Foodborne illness acquired in the United States-Major pathogens. Emerg Infect Dis 2011, 17:7-15.

Shimizu A, Fujita M, Igarashi H: Characterization of Staphylococcus aureus Coagulase Type VII Isolates from S taphylococcal Food Poisoning Outbreaks (1980 - 1995) in Tokyo, Japan, by Pulsed-Field Gel Electrophoresis. J Clin Microbiol 2000, 38:3746-3749.

Swaminathan B, Gerner-Smidt P: The epidemiology of human listeriosis. Microbes Infect 2007, 9:1236-1243.

Switt AIM, Soyer Y, Warnick LD, Wiedmann M: Emergence, distribution, and molecular and phenotypic characteristics of Salmonella enterica serotype 4,5,12:i:-.Foodborne Pathog Dis 2009, 6:407-415.

Talbot E a, Gagnon ER, Greenblatt J: Common ground for the control of multidrug-resistant Salmonella in ground beef.Clin Infect Dis 2006, 42:1455-1462.

Vimont a., Vernozy-Rozand C, Delignette-Muller ML: Isolation of E. coli O157:H7 and non-O157 STEC in different matrices: Review of the most commonly used enrichment protocols. Lett Appl Microbiol 2006, 42:102-108.

Williams AG, Withers SE: Microbiological characterisation of artisanal farmhouse cheeses manufactured in Scotland. Int J Dairy Technol 2010, 63:356-369. 